\title{
Some Observations on Grid Convergence
}

Manuel D. Salas

NASA Langley Research Center

Hampton, VA 23681-2199

\section{CORRESPONDENCE:}

Manuel D. Salas

NASA Langley Research Center

Mail Stop 499

Hampton, VA 23681-2199

Phone: 757-864-4611

FAX: 757-864-8469

e-mail: m.d.salas@nasa.gov 


\title{
Some Observations on Grid Convergence \\ Manuel D. Salas \\ NASA Langley Research Center \\ Hampton, VA 23681-2199
}

\begin{abstract}
It is claimed that current practices in grid convergence studies, particularly in the field of external aerodynamics, are flawed. The necessary conditions to properly establish grid convergence are presented. A theoretical model and a numerical example are used to demonstrate these ideas.
\end{abstract}

Keywords: grid convergence, numerical accuracy, code verification, Ringleb flow, drag prediction, external aerodynamics.

\section{Introduction}

Grid convergence is an important process in verifying that discrete numerical solutions are valid representations of the governing partial differential equations describing the phenomenon under investigation. With increased emphasis on the subject of Verification and Validation [1], [2] and with many fluid and numerical methods journals now adopting policy statements on numerical accuracy, grid convergence is a topic frequently visited, but rarely performed correctly. In this note, the state-of-the-practice of computational fluid dynamics (CFD), particularly as it is applied for external aerodynamics, is reviewed and some observations are made regarding the apparent lack of convergence. 
The results presented are for regular-structured grids. Similar results can be obtained for regular-unstructured grids (i.e. grids consisting of isosceles triangles in 2D). The general unstructured grid problem has not been investigated.

\section{Current Practice}

To illustrate the state-of the-practice in grid convergence, consider the drag prediction workshops sponsored by the Applied Aerodynamics Technical Committee of the American Institute of Aeronautics and Astronautics. The first of these workshops, the Drag Prediction Workshop-I (DPW-I), was held in June of 2001. The workshop required the evaluation of the force and moment coefficients by CFD of a relatively simple wingbody configuration known as the DLR-F4. This particular configuration was chosen because it had been tested in 3 wind tunnels. A total of 35 solutions based on Reynolds averaged Navier-Stokes (RANS) flow solvers, representing a very wide cross section of the CFD community, were presented at the workshop. Of these, 21 solutions were obtained on grids provided by the workshop organizers and 14 on grids created by the participants. A summary of the data presented at the workshop is given in [3] and a statistical analysis of the data is presented in [4]. Surprisingly, a demonstration of grid convergence was not a requirement of the workshop. However, in a later study LeeRausch et al. [5] assessed the grid convergence of 4 of the codes used in the workshop using the same DLR-F4 configuration. The second Drag Prediction Workshop-II (DPWII) was held in June of 2003. The DPW-II Organizing Committee, at the request of the workshop participants, focused the second workshop on a more complex model [5]. The 
new model, the DLR-F6, consisted of wing-body (WB) and wing-body nacelle-pylon (WBNP) configurations. The DLR-F6 wing-body configuration was very similar to the DLR-F4 configuration with minor changes in the airfoil sections introduced to reduce the chances of boundary layer separation. Wind tunnel tests for this configuration were conducted in 1990 at the S2MA pressurized tunnel of the ONERA center at Modane. As in the first workshop, standard grids were provided, but for this workshop a grid refinement study was required. A total of 29 data sets were submitted, however a number of these represented variations of the same code. The solutions presented at this workshop are summarized in [6] and were statistically analyzed in [7]. Additional grid convergence studies of the DLR-F6 configurations are presented in [8] and [9].

A review of the references cited above, and of the many other papers associated with the drag prediction workshops, reveals some curious facts. The current practice is to conduct convergence studies on the basis of surface integral coefficients such as the coefficients of drag, lift and moment. These are plotted with respect to some measure of the grid size, which we shall call $h$, raised to a power $p$, where $p$ is the formal grid accuracy of the numerical scheme used. Finally, we notice that it is common practice in the literature to present grid convergence studies that do not show convergence. Hence, we have to conclude that the actual act of doing a grid convergence study far outweighs the results of the study. Figure 1 is representative of a typical figure from these studies. Here the moment coefficients from four of the codes used in the DPW-II are plotted against the grid spacing squared, since the methods used are formally second order accurate. 
The practice of evaluating grid convergence with respect to $h^{p}$ follows from usual Taylor expansion studies of a numerical scheme which show that a computed quantity $f_{c}$ is related to the exact value $f_{e}$ of the solution of the differential equation by

$$
f_{e}=f_{c}+c h^{p}+\text { H.O.T. },
$$

where $c$ is a coefficient independent of $h$. For $h$ sufficiently small, the higher order terms are negligible compared to the leading term $h^{p}$ and the exact solution is related to the computed solution simply by

$$
f_{e} \approx f_{c}+c h^{p} .
$$

When this happens, we say that the solutions are in the asymptotic range of convergence. If we accept the validity of this expression and we have solutions on three grids which have converged in time (or iteration parameter) it is unnecessary to assume that $p$ corresponds to the formal order of accuracy, since given three solutions Eq. (1.2) can be solved for the three unknowns: $f_{e}, c$ and $p$. If we evaluate these quantities from the data reported in the DPW-II (WB case), see Table III of [10], we find that $p$ is far from its formal value of two. For example, if we consider the data in Fig. (1), for the third code we find $p=8.54$ and for the other three codes a real solution doesn't exist, since the behavior of the moment coefficient is not monotonic with $h$. It is tempting to dismiss Eq. 
(1.2) by suggesting that the grids used in these studies were not sufficiently fine and therefore the solutions are not in the asymptotic range. However, we believe that something more subtle is at work.

\section{The $h$ Condition}

For two and higher dimensional problems, Eq. (1.1) is a simplification of the actual Taylor expansion. For a two-dimensional problem the expansion reads

$$
f_{e}=f_{c}+a h_{x}^{p}+b h_{y}^{p}+\text { H.O.T. }
$$

where $h_{x}$ and $h_{y}$ are the grid spacing in the $x$ and $y$ directions, respectively, and $a, b$ are coefficients independent of $h_{x}$ and $h_{y}$. Note that if the grid is not uniform in the physical space, we assume an invertible one to one mapping to a computational space that renders the grid spacing uniform. If that is the case, Eq. (1.3) represents the expansion in the computational space. For both $h_{x}$ and $h_{y}$ sufficiently small, we have

$$
f_{e} \approx f_{c}+a h_{x}^{p}+b h_{y}^{p}
$$

The current practice is to replace (1.4) with (1.2) by defining ${ }^{1}$

\footnotetext{
${ }^{1}$ For a three-dimensional problem $h$ is defined as $h=\left(h_{x} h_{y} h_{z}\right)^{1 / 3}$.
} 


$$
h=\sqrt{h_{x} h_{y}} .
$$

Equation (1.5) defines $h$ to be the side of the square with area equal to the rectangle with sides $h_{x}, h_{y}$. The definition of $h$ is not unique. Among others, $h$ could be defined as the diagonal: $h=\sqrt{h_{x}^{2}+h_{y}^{2}}$.

There is an obvious advantage in using Eq. (1.2) instead of Eq. (1.4), since there are four unknowns in (1.4) and only three in (1.2), but this advantage comes with a condition. To be able to replace (1.4) by (1.2) it is necessary that

$$
\frac{h_{y, k}}{h_{x, k}}=\chi \quad \text { for } \forall k, k=1,2,3, \ldots, K
$$

where $\chi$, the grid aspect ratio, is constant over all $k$-grids ${ }^{2}$.

An example will illustrate this condition. Assume that we have a problem for which we know the exact solution and all other relevant information. Say that we know that $a=1$, $b=5, p=2, f_{e}=1$ and $h$ is defined by (1.5). If we solve the problem on three grids that satisfy (1.6), we find the results listed on Table I. On the table, $\varepsilon$ is the error defined by: $\varepsilon=f_{e}-f_{c}$, and $N_{x}$ and $N_{y}$ are the number of grid points in the $x$ and $y$ directions,

\footnotetext{
${ }^{2}$ For a three-dimensional problem $\frac{h_{y, k}}{h_{x, k}}=\frac{h_{z, k}}{h_{x, k}}=\chi$ for $\forall k, k=1,2,3, \ldots, K$.
} 
respectively. If we now use the computed $f_{c, k}$ and the values of $h_{k}$ in Eq. (1.2), we find $p=2, f_{e}=1$ and $c=12.9$, which is consistent with the original data. Now we repeat the exercise using three grids that do not have constant aspect ratio. The results are listed in Table II. If we use these results $\left(f_{c, k}, h_{k}\right)$ in Eq. (1.2), we find $p=2.36, f_{e}=.999$ and $c=34.75$. The results are no longer consistent with the original data. It is important to note that the data in Table II represents data of a second order accurate method, but because the grid aspect ratio is not constant Eq. (1.2) is unable to recover this fact. Note also that the grid points in Table II were chosen such that the ratio of successive $h_{k}$ 's is constant, but this, despite claims in [2], does not make Eq. (1.2) valid.

\section{A Numerical Example: Ringleb Flow}

Ringleb flow describes an inviscid, compressible, transonic flow, with isentropic exponent of 1.4 , making a $180^{\circ}$ turn. The exact solution for this flow was found by Ringleb [11] in 1941. We solve the Ringleb flow problem using MacCormack's [12] second order accurate, two step method on grids consisting of streamlines and isopotential lines, as depicted in Figure 2. The lower boundary for the case we will be computing corresponds to a streamline that reaches a Mach number of 1.8 at $x=0$ and extends to the point where its Mach number is 0.6 . The upper boundary corresponds to a streamline that reaches a Mach number of 1.2 at $x=0$ and is terminated by the isopotential line emanating from the lower boundary. Since the flow is symmetric about $x=0$, we only compute in the half-plane $x \leq 0$. We consider 5 grid-sets, identified by an 
index $i$, for each grid-set the grid aspect ratio is constant. Each grid-set consists of 4 grids identified by an index $k$. For each grid we compute a pressure-residual to establish temporal convergence and an error based on the magnitude of the flow velocity defined by

$$
\varepsilon=\sum_{m=1}^{M} \sum_{n=1}^{N}\left|q_{\text {exact }}-q_{n, m}\right| /(N \times M),
$$

where $q_{\text {exact }}$ is the exact magnitude of the flow velocity, $q_{n, m}$ is the computed magnitude of the flow velocity at grid point $n, m$ and $N, M$ are the total number of grid points in the streamline and iso-potential line directions, respectively. The results are tabulated in Table III.

In Figure 3 we plot the $\log$ of $\varepsilon v s$. the $\log$ of $h$. The slope of the lines $\chi=$ constant corresponds to the convergence rate $p$. We notice that for grid-sets 1 and 2, corresponding to $\chi(.5,1)$, the four grid values line-up in a straight line. Grid-set $3, \chi=2$, deviates slightly from a straight line, while the deviation is more pronounced for grid-sets 4 and $5, \chi(3,4)$. The significance of this is that as the grid aspect ratio increases a finer grid is needed to enter the asymptotic range. Thus, grid $i=1, k=1$ is in the asymptotic range, but grid $i=4$, $k=1$ is not. The last column of Table III tabulates the convergence rate $p$ for each grid-set based on the slope of the two finest grids on each grid-set. The value of $p$ rounds-up to 2.2 for the first 3 grid-sets then slightly increases for the last two grid-sets, an indication that finer grids are needed to enter the asymptotic range as $\chi$ increases. This is shown on 
Figure 4 where the convergence rate is plotted as a function of $h$. For this figure $p$ is obtained from

$$
p=\left(\log \left(\varepsilon_{k}\right)-\log \left(\varepsilon_{k-1}\right)\right) /\left(\log \left(h_{k}\right)-\log \left(h_{k-1}\right)\right),
$$

and $h$ follows from

$$
h=\left(h_{k}+h_{k-1}\right) / 2 .
$$

Calculations with finer grids than those listed on Table III were used to create this figure. We also notice in Figure 3 a folding-over of grid-sets 3 and 4. This is an indication that with $h$-fixed there is a $\chi$ that minimizes the error. This is depicted on Figure 5 where $\chi$ is unfolded by plotting the error $v s$. the grid aspect ratio while keeping $h$ constant at 0.0408 . For this problem, the optimum $\chi$ has a value approximately equal to 2.25. Figure 6 shows the error $\varepsilon$ as a function of $h^{p}$ with $p=2.2$. Only the three finest grid $(k=2,3,4)$ values are used in this figure and the error is extrapolated for $h \rightarrow 0$. These results show that Eq. (1.2) is valid when condition (1.6) is satisfied. What happens if this is not the case? Consider the following grid-set $(i, k)=(1,1),(3,3)$ and $(4,3)$. This set consists of grids with different aspect ratios, but the error is monotonic with $h$. Although the data from this set corresponds to solutions with convergence rate of approximately 2.2 , when we compute the convergence rate solving Eq. (1.2) we find $p=9.94$. If the grids do not satisfy condition(1.6), then Eq. (1.2) cannot be used to establish convergence. However, while it is convenient to use Eq. (1.2) it is not necessary to do so. Using the values from grids $(i, k)=(1,4),(2,4)$ and $(3,4)$, which clearly do not satisfy $(1.6)$, we find from Eq. $(1.4)$ that 
$p=2.4$. For this grid-set the error is not monotonic with $h$ and if $p$ is evaluated by solving

Eq. (1.2), we find that $p$ is not real.

\section{Concluding Remarks}

Today grid convergence studies with grids in the tens of million grid points are carried out to assess and verify the validity of numerical solutions for flows over aircraft configurations. In the very near future grids in the hundreds of million grid points will be used. These studies are expensive, time consuming and impose large demands on our computer infrastructure. Many of these previous grid convergence studies have been flawed, most probably not because of problems with the codes, but because the procedures used to establish convergence have been flawed. A grid convergence study has two primary purposes: 1) to establish convergence, and 2) to determine the rate of convergence. The first is significantly more important than the second. As part of a convergence study, we need to determine that the solutions are in the asymptotic range and we need to find what the actual convergence rate is. Neither should be assumed as part of the process. In establishing the actual convergence rate, it is important to remember that the formal rate is established on the basis of a numerical algorithm that is used for the calculation of the field points. The algorithm is usually modified at the boundaries and boundary data alone might not reflect the formal rate. ${ }^{3}$ Finally, if we want to take advantage of the simplifications inherent in (1.2), it is necessary to insure that the grids used conform to condition (1.6).

\footnotetext{
${ }^{3}$ This was not the case in our example with Ringleb flow. Using only boundary data we obtained convergence rates close to the rates we found using the field data. However, much care was taken in the implementation of the boundary conditions which resulted in a formally second order method at the boundaries.
} 


\section{References}

[1] Roache, P. J. Verification and Validation in Computational Science and Engineering, Hermosa Publishers, New Mexico; 1998.

[2] Oberkampf, W. L. and Trucano, T. G. Verification and Validation in Computational Fluid Dynamics, Progress in Aerospace Science, 2002; 38:210272.

[3] Levy, D.W., Zickuhr, T., Vassberg, J., Agrawal, S., Wahls, R. A., Pirzadeh, S. and Hemsch, M. J. Summary of Data From the First AIAA CFD Drag Prediction Workshop, AIAA Paper No. 2002-0841, 2002.

[4] Hemsch, M. J., Statistical Analysis of CFD Solutions From the Drag Prediction Workshop, AIAA Paper No. 2002-0842, 2002.

[5] Lee-Rausch, E. M., Buning, P. G., Mavriplis, D. J., Morrison, J. H., Park, M. A., Rivers, S. M. and Rumsey, C. L. CFD Sensitivity Analysis of a Drag Prediction Workshop Wing/Body Transport Configuration, AIAA Paper No. 2003-3400, 2003.

[6] Laflin, K. R., Klausmeyer, S. M., Zickuhr, T., Vassberg, J. C., Wahls, R. A., Morrison, J. H., Brodersen, O., Rakowitz, M., Tinoco, E. N. and Godard, J. L. Summary of Data From the Second AIAA CFD Drag Prediction Workshop, AIAA Paper No. 2004-555, 2004.

[7] Hemsch, M. J. and Morrison, J. H. Statistical Analysis of CFD Solutions from $2^{\text {nd }}$ Drag Prediction Workshop, AIAA Paper No. 2004-556, 2004. 
[8] Rumsey, C. L., Rivers, S. M., and Morrison, J. H. Study of CFD Variation on Transport Configurations From the Second Drag-Prediction Workshop, AIAA Paper No. 2004-0394, 2004.

[9] Lee-Rausch, E. M., Frink, N. T., Mavriplis, D. J., Rausch, R. D. and Milholen, W. E. Transonic Drag Prediction on a DLR-F6 Transport Configuration Using Unstructured Grid Solvers, AIAA Paper No. 2004-0554, 2004.

[10] Salas, M. D. Digital Flight: The Last CFD Aeronautical Grand Challenge, JSC to appear, 2005.

[11] Ringleb, F. Exacte Lösungen der Differentialgleichunsen eineradiabatischen Gasströmung, ZAMM 1941;20:185-198.

[12] MacCormack, R. W. The Effect of Viscosity in Hypervelocity Impact Cratering, AIAA Paper No. 69-354, 1969. 


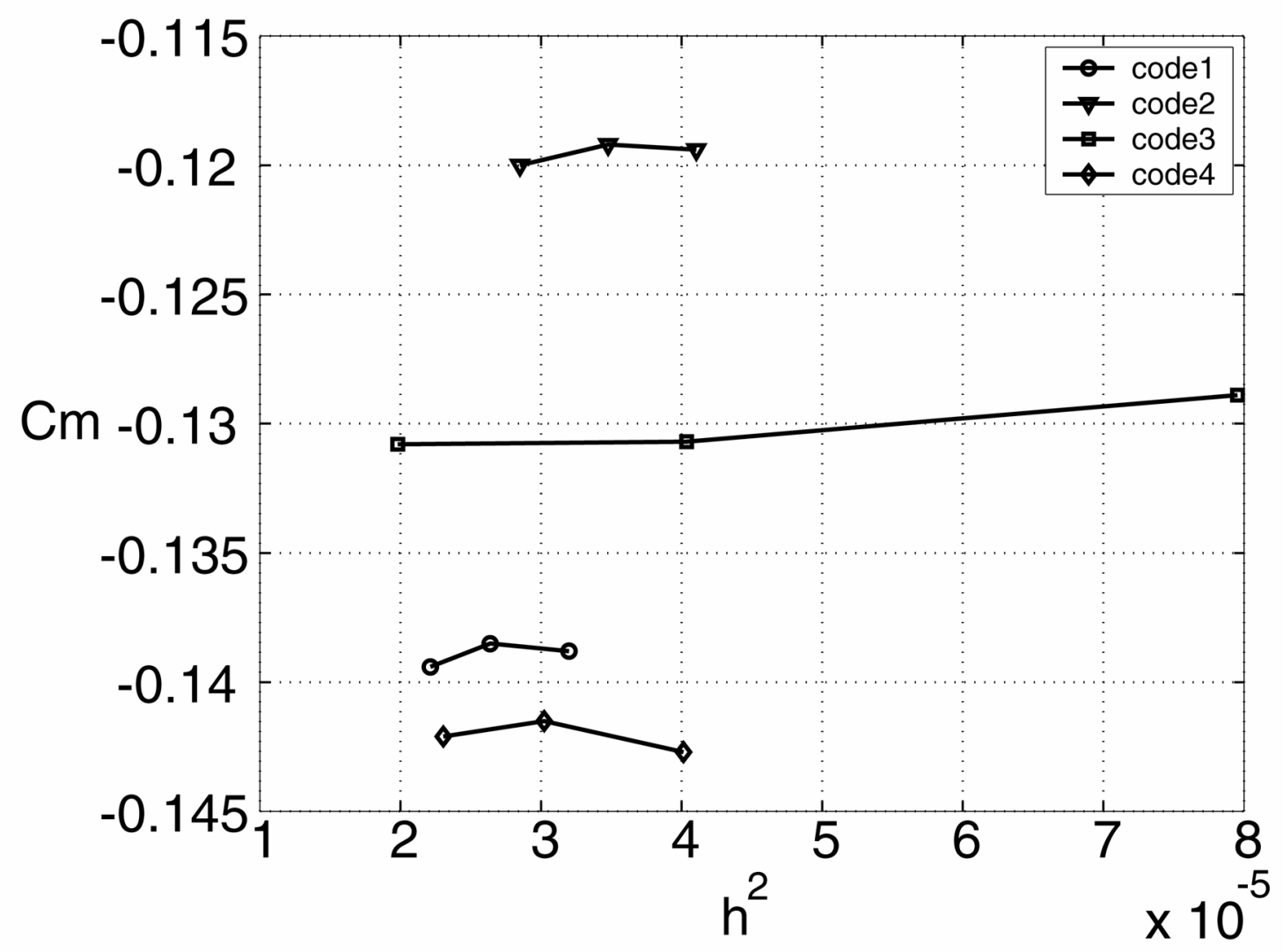

Figure 1. Variation of moment coefficient as a function of grid spacing squared. Data from DPW-II, WB case. 


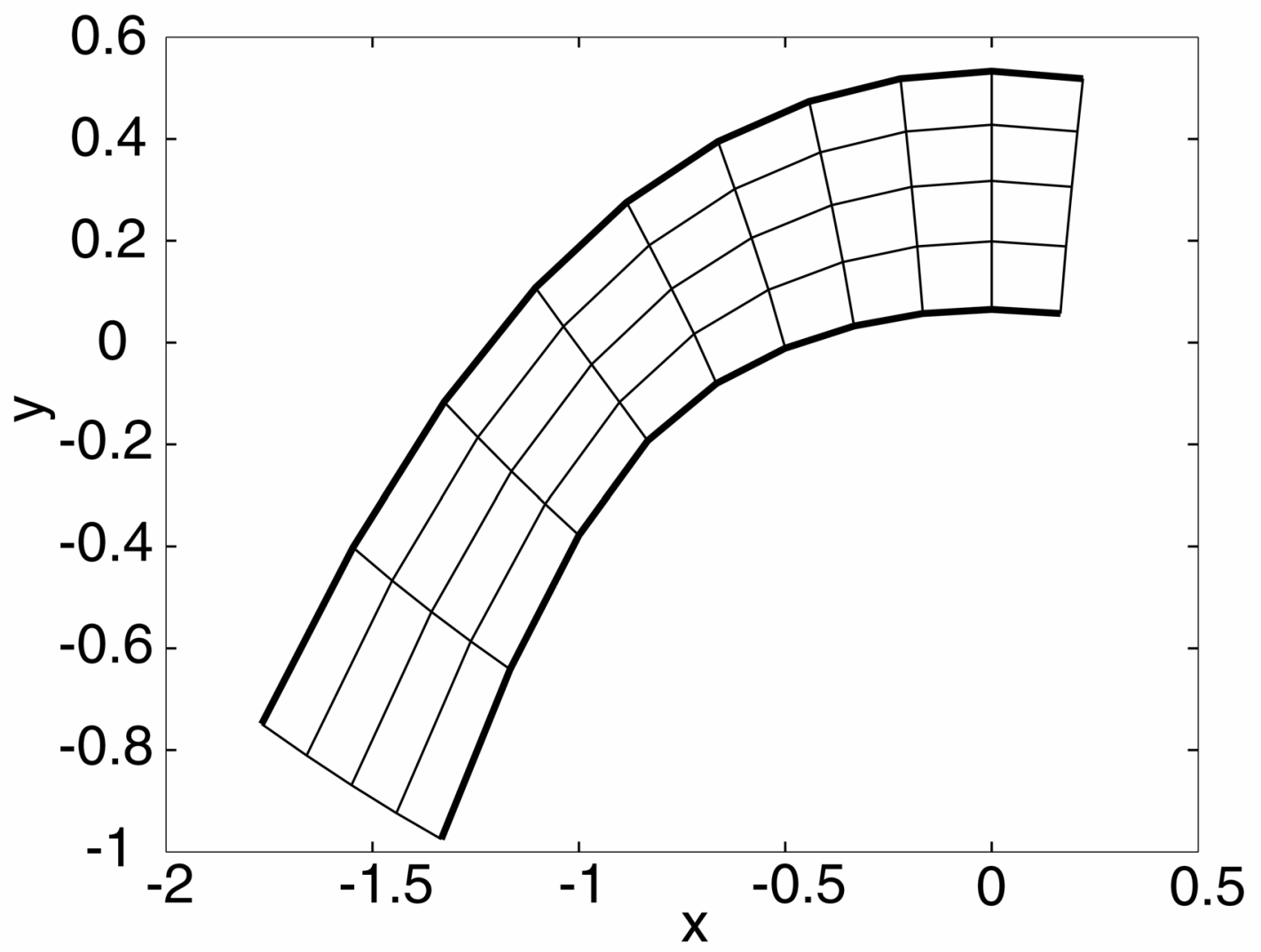

Figure 2. Typical grid used in numerical calculations of Ringleb flow. 


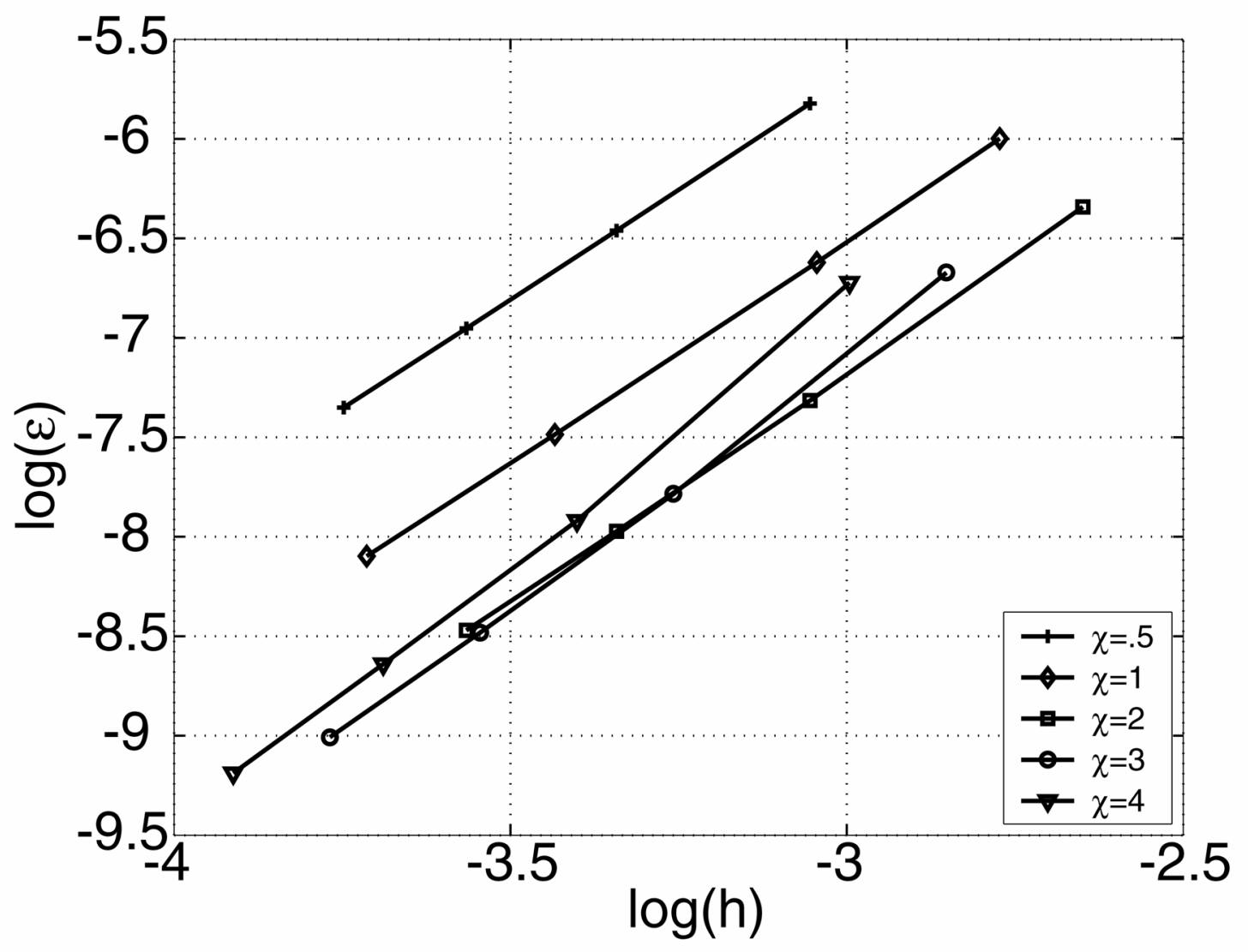

Figure 3. $\log (\varepsilon)$ vs. $\log (h)$ for several grid aspect ratios. 


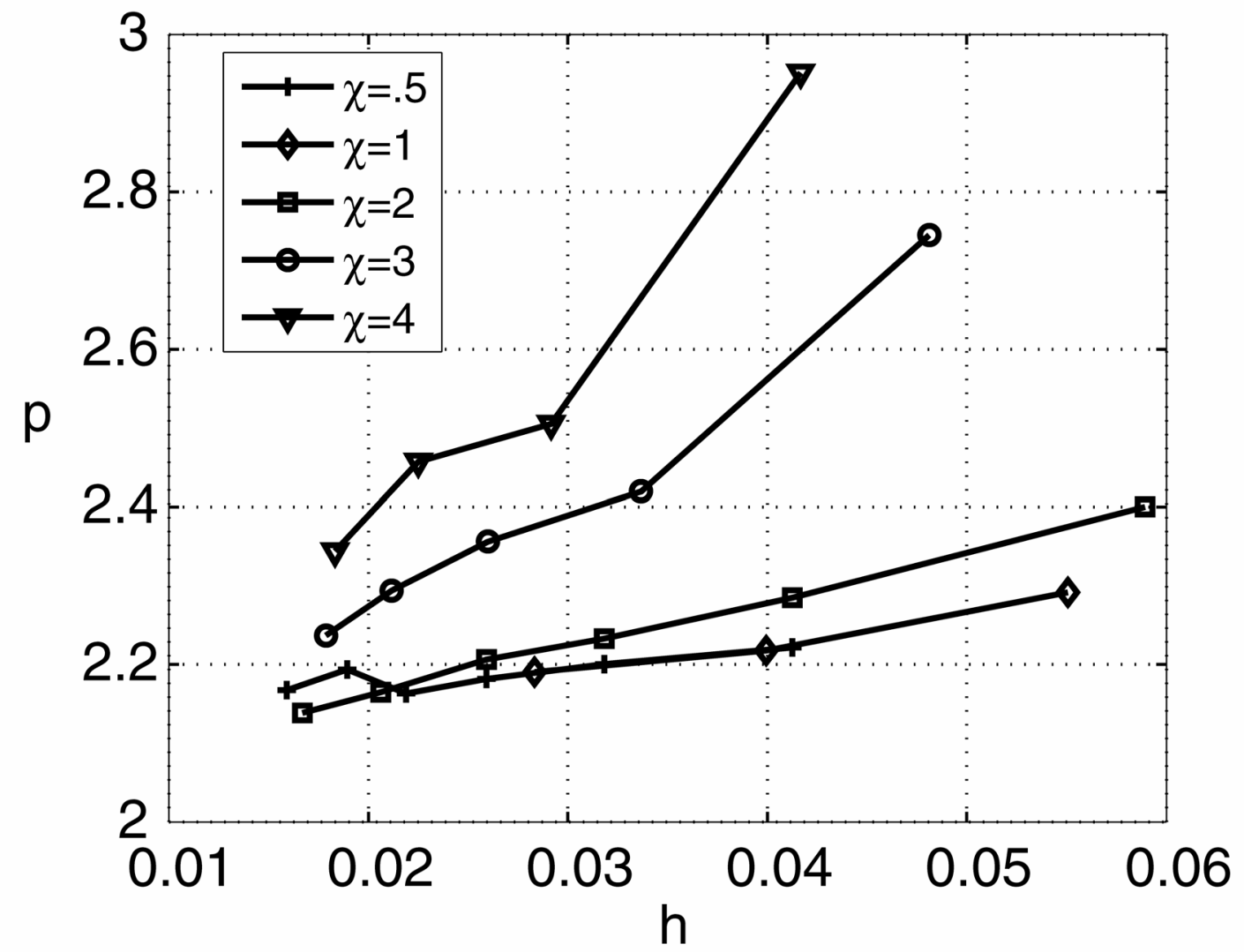

Figure 4. Convergence rate behavior with grid refinement. 


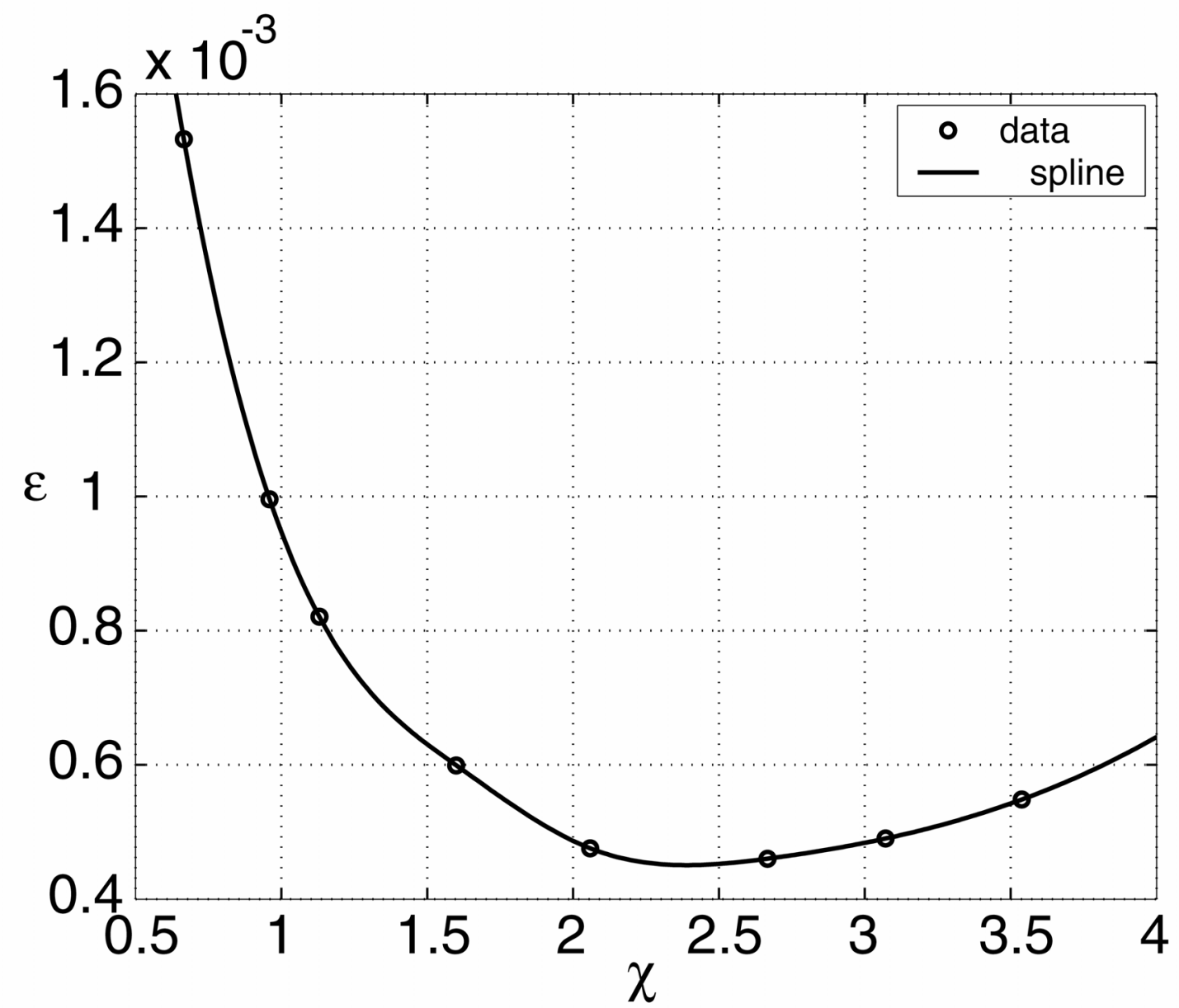

Figure 5. Error vs. grid aspect ratio with $h$ constant at 0.0408 . 


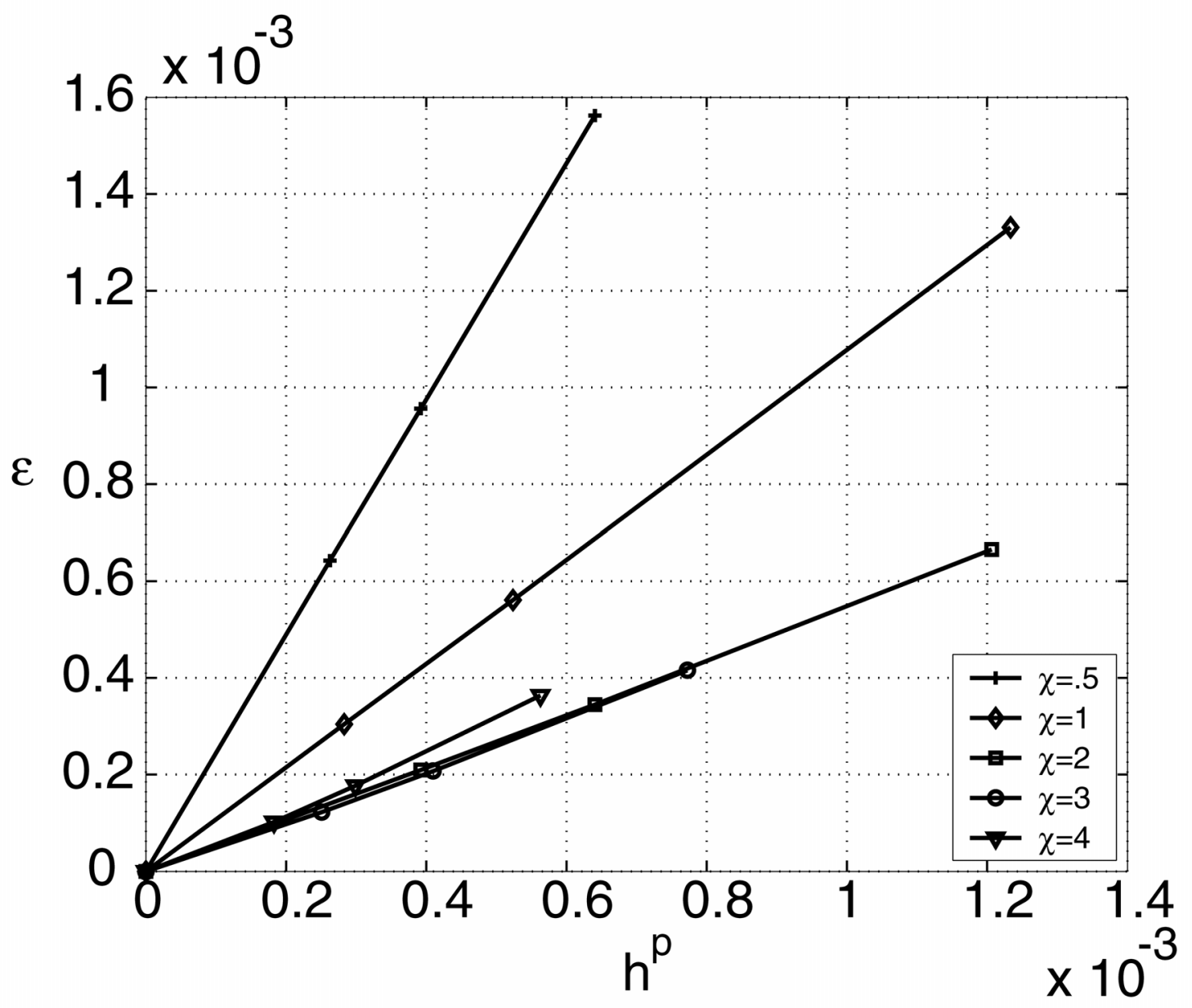

Figure 6. Computed Ringleb flow error vs. $h^{p}$ with $p=2.2$. 


\begin{tabular}{ccccccc}
\hline $\boldsymbol{k}$ & $\boldsymbol{N}_{\mathbf{x}}$ & $\boldsymbol{N}_{\boldsymbol{y}}$ & $\chi$ & $\boldsymbol{h}$ & $\boldsymbol{\varepsilon}$ & $f_{c}$ \\
\hline \hline 1 & 25 & 10 & 2.5 & .06324 & .0516 & .9484 \\
2 & 50 & 20 & 2.5 & .03162 & .0129 & .9871 \\
3 & 75 & 30 & 2.5 & .02108 & .0057 & .9943 \\
\hline
\end{tabular}

Table I. Results from a simulation with constant grid aspect ratio. 


\begin{tabular}{ccccccc}
\hline $\boldsymbol{k}$ & $\boldsymbol{N}_{\mathbf{x}}$ & $\boldsymbol{N}_{\boldsymbol{y}}$ & $\chi$ & $\boldsymbol{h}$ & $\boldsymbol{\varepsilon}$ & $f_{c}$ \\
\hline \hline 1 & 25 & 10 & 2.5 & .06324 & .0516 & .9484 \\
2 & 40 & 20 & 2.0 & .03535 & .0131 & .9869 \\
3 & 64 & 40 & 1.6 & .01976 & .0034 & .9966 \\
\hline
\end{tabular}

Table II. Results from a simulation with varying grid aspect ratio. 


\begin{tabular}{cccccccc}
\hline $\boldsymbol{i}$ & $\boldsymbol{k}$ & $\boldsymbol{N}$ & $\boldsymbol{M}$ & $\begin{array}{c}\chi= \\
\boldsymbol{N} / \boldsymbol{M}\end{array}$ & $\boldsymbol{\varepsilon}$ & $\begin{array}{l}\text { pressure } \\
\text { residual }\end{array}$ & $\boldsymbol{p}$ \\
\hline \hline 1 & 1 & 15 & 30 & .5 & 0.0029603 & $6.55 \mathrm{e}-007$ & 2.1815 \\
& 2 & 20 & 40 & .5 & 0.0015623 & $9.44 \mathrm{e}-008$ & \\
& 3 & 25 & 50 & .5 & 0.0009561 & $3.06 \mathrm{e}-008$ & \\
& 4 & 30 & 60 & .5 & 0.0006423 & $1.47 \mathrm{e}-008$ & \\
2 & 1 & 16 & 16 & 1 & 0.0024823 & $1.65 \mathrm{e}-010$ & 2.1905 \\
& 2 & 21 & 21 & 1 & 0.0013311 & $1.91 \mathrm{e}-007$ & \\
& 3 & 31 & 31 & 1 & 0.0005612 & $2.14 \mathrm{e}-008$ & \\
& 4 & 41 & 41 & 1 & 0.0003042 & $3.80 \mathrm{e}-009$ & \\
3 & 1 & 20 & 10 & 2 & 0.0017611 & $1.98 \mathrm{e}-008$ & 2.2330 \\
& 2 & 30 & 15 & 2 & 0.0006655 & $3.48 \mathrm{e}-008$ & \\
& 3 & 40 & 20 & 2 & 0.0003449 & $1.68 \mathrm{e}-008$ & \\
& 4 & 50 & 25 & 2 & 0.0002095 & $3.94 \mathrm{e}-009$ & \\
4 & 1 & 30 & 10 & 3 & 0.0012655 & $1.46 \mathrm{e}-008$ & 2.3558 \\
& 2 & 45 & 15 & 3 & 0.0004158 & $5.68 \mathrm{e}-010$ & \\
& 3 & 60 & 20 & 3 & 0.0002072 & $3.72 \mathrm{e}-011$ & \\
& 4 & 75 & 25 & 3 & 0.0001225 & $2.69 \mathrm{e}-011$ & \\
5 & 1 & 40 & 10 & 4 & 0.0012033 & $1.45 \mathrm{e}-010$ & 2.4573 \\
& 2 & 60 & 15 & 4 & 0.0003636 & $5.55 \mathrm{e}-011$ & \\
& 3 & 80 & 20 & 4 & 0.0001768 & $1.02 \mathrm{e}-011$ & \\
& 4 & 100 & 25 & 4 & 0.0001022 & $1.00 \mathrm{e}-011$ & \\
\hline
\end{tabular}

Table III. Cases computed for Ringleb flow. 